\title{
Miliary Tuberculosis in a Healthy Adult
}

\author{
Tatiana Denega MD, David Griffith MD
}

\begin{abstract}
Miliary tuberculosis (TB) is a consequence of mycobacterial dissemination. In the early stages it can be a challenging diagnosis due to the systemic but non-specific symptoms and subtle and gradual imaging changes. The dissemination of wall components of Mycobacterium tuberculosis can induce a severe inflammatory response with generalized endothelial cell damage, leading to fatal complications, such as disseminated intravascular coagulation (DIC) and acute respiratory distress syndrome (ARDS). We report a 34-year-old woman with no known medical disorders. She was diagnosed with miliary TB at the late stage of infection which presented with a fulminant clinical course, including DIC and multi-organ failure. The autopsy revealed multiple foci of infection in the kidneys, spleen, meninges, lungs, and vertebral bodies. Dissemination of TB to extrapulmonary organs is a diagnostic challenge when the onset precedes pulmonary symptoms, thereby eluding clinical suspicion and a rapid diagnosis. The duration from the onset of symptoms and laboratory abnormalities can be key predictors for the development of late stage complications. The poor prognosis of military TB can be mitigated by the early detection and rapid initiation of appropriate antituberculous medications.
\end{abstract}

Key words: miliary tuberculosis, disseminated intravascular coagulation, acute respiratory distress syndrome

\section{INTRODUCTION}

Disseminated tuberculosis (TB) is characterized by the spread of Mycobacterium tuberculosis to various extrapulmonary organs with the formation of caseous tubercules. Generally, the lungs are the primary source of bacterialacquisition which may be followed by hematogenous and lymphatic dissemination of the bacilli. In essentially all instances, tuberculosis is considered a systemic disease as dissemina-

Corresponding author: Tatiana Denega MD Contact Information: tdenega@yahoo.com DOI: 10.12746/swrccc2014.0205.061 tion of the bacteria by these routes occurs routinely. However, in most instances, the tuberculous foci are contained by a vigorous immune response, and the disease presents only as a lung infection. Clinically significant TB dissemination occurs in the setting of progressive primary TB infection in relatively or absolutely immunocompromised hosts, such as infants and young children or patients with acquired immune deficiency syndrome (AIDS). Additionally, TB disseminationmay follow the reactivation of initial immune TB containment after initial infection and establishment of latent tuberculous infection (LTBI) interrupted by some alteration in immune surveillance, such as 
with a patient with LTBI placed on a tumor necrosis factor alpha blocker. For some patients, such as the one presented in this report, TB dissemination may occur with no identifiable predisposition. Suspecting disseminated TB and including it in a differential diagnosis may be challenging when the patient is not known to be immunocompromised and has no history of $L T B I$ and no obvious risk factors for acquiring TB. The symptoms of extrapulmonary TB are also generally not specific for TB and may precede more typical pulmonary TB symptoms adding more complexity to the diagnosis.

\section{Case Presenttion}

A 34-year-old Caucasian woman was referred to the phthisiopulmonology clinic in Moscow, Russia, with severe dyspnea at rest, cough with scant sputum, fever, generalized weakness, and loss of appetite. The patient was in her usual health until approximately seven weeks earlier when she began to exhibit dyspnea on exertion that she attributed to her weight, which was approximately $180 \mathrm{lbs}$ at the onset of her symptom. Dyspnea persisted despite the three weeks of dieting. The patient recalled that at this time she begun to produce scant, clear, mucoid sputum on cough, noted an elevation of temperature in the evenings $\left(100.4^{\circ} \mathrm{F}\right)$, and had malaise. She reported no rhinorrhea, sore throat, wheezing, chills, dizziness, palpitations, or orthopnea. Increasing dyspnea, malaise, and fever prompted the patient to seek treatment at the end of the fourth week of symptoms, and she was hospitalized at the municipal hospital for the treatment of suspected pneumonia. Two weeks of treatment with ceftriaxone and azithromycin yielded no improvement. Her temperature continued to increase, and her dyspnea worsened. Three days before the admission to the phthisiopulmonology clinic, the patient was consulted by a phthisiatrician due to progressive worsening of her symptoms. Three acid fast stain microscopies of sputum were performed, and all three were negative. Despite these results, miliary TB was suspected, and she was referred to the phthisiopulmonology clinic.
The past medical history revealed that the patient had back pain for five years and had been under the care of a traumatologist with little improvement. A year prior to her presentation outlined above, the patient was diagnosed with a compression fracture in the thoracic spine but refused treatment (Figure 1). She worked as a lawyer, was married, and had three children. She lived with her family in a three bedroom apartment in Moscow. She did not travel in the prior year. She had no known allergies, did not smoke, drink alcohol, or use illicit drugs. She had no known tuberculosis contacts; her tuberculin skin test status was unknown. She received a BCG vaccination at birth.

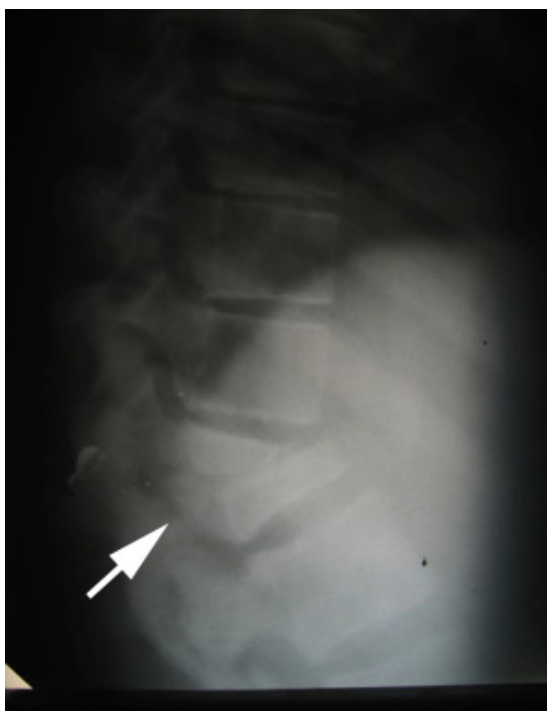

Figure 1. Thoracic spine radiograph showing a vertebral compression fracture at the level of T11-12 vertebral bodies.

On examination, she was oriented in person, place, and time. She weighed $156 \mathrm{lbs}$ and measured $158 \mathrm{~cm}$ in height. The pulse was 140 beats per minute, the temperature $102.2^{\circ} \mathrm{F}$, and the respiratory rate 36 breaths per minute. Facial cyanosis was noted. No cervical, axillary, epitrochlear, inguinal, or popliteal lymphadenopathy was noted. Lungs examination revealed bronchial breath sounds throughout both lung fields and dullness to percussion in both bases. 
There was no jugular vein distention. Heart sounds were normal without murmurs or rubs. The abdomen was soft with apalpable liver tip $2 \mathrm{~cm}$ below the costal margin. She had 1+ bilateral lower extremity edema.

On admission her oxyhemoglobin saturation was $87 \%$ while breathing ambient air. The peripheral blood white cell count was 9300 cells per cubic millimeter with $4 \%$ lymphocytes, hemoglobin $11.7 \mathrm{~g} /$ $\mathrm{dL}$, ESR level $27 \mathrm{~mm}$ per hour, blood urea nitrogen level $189 \mathrm{mg} / \mathrm{dL}$, creatinine level $3.3 \mathrm{mg} / \mathrm{dL}$, bilirubin normal, and hepatic transaminases normal. Urine analysis revealed specific gravity 1.015 , protein $3+$, and white blood cells $20-30$ cells per LPF. She was HIV seronegative. Microscopy of sputum smears and culture were not collected due to the patient's scant sputum production and inability to tolerate sputum induction because of severe dyspnea.

The initial chest radiograph from the municipal hospital (Figure 2) indicated uniform 1 to $2 \mathrm{~mm}$ diameter diffuse miliary densities without frank consolidation. A new chest radiograph was performed on the first day of admission to the phthisiopulmonolgy clinic, which depicted miliary densities with areas of enlarged and coalesced densities forming patchy consolidation.

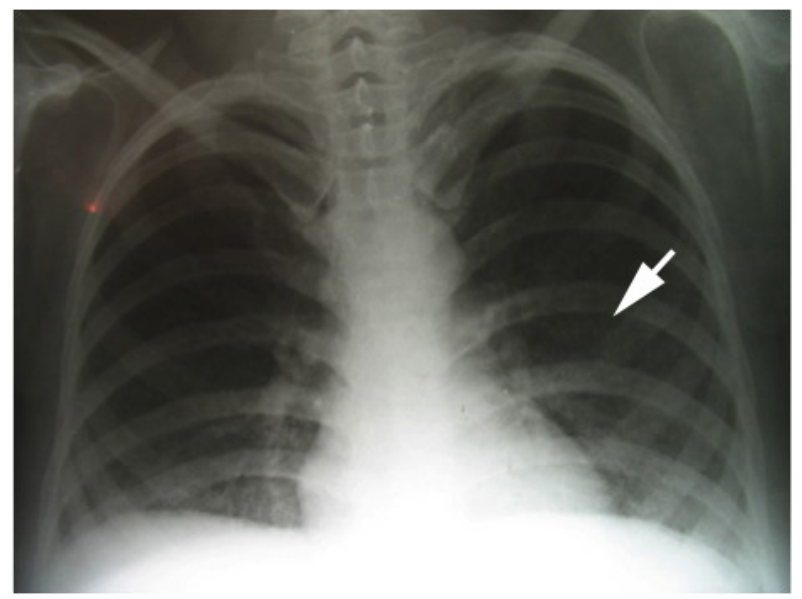

Figure 2. Initial chest radiograph showing diffuse, bilateral miliary densities.
At the phthisiopulmonology clinic on day one, the patient was placed on intravenous isoniazid 300 $\mathrm{mg}$, rifampicin $450 \mathrm{mg}$, pyrazinamide $1500 \mathrm{mg}$, and intramuscular pyridoxine $25 \mathrm{mg}$ twice a day. On day two, the patient remained febrile and dyspneic; her hypoxemia became progressively more severe. The patient became confused and somnolent and required endotracheal intubation and mechanical ventilation. The patient developed evidence of DIC with bleeding from the endotracheal tube and urinary catheter. The bleeding stopped aftertransfusion of four units of fresh frozen plasma. On day three, the patient died due to respiratory insufficiency and multi-organ system failure.

An autopsyrevealed numerous miliary foci in the lungs (Figure 3), spleen, pia meninges of the cerebellum, destruction of the bodies of thoracic vertebrae 11-12 with paravertebral abscess formation (Figure 4), cavernous destruction of the left kidney (Figure 5) and pyonephrosis of the right kidney. Biopsy specimens were taken from the pia meninges, lungs, spleen, thoracic vertebrae 11-12, paravertebral abscess, and both kidneys. Analysis of the biopsy specimens revealed characteristic granulomatous inflammation with caseous necrosis. All acid fast stains of biopsy tissues listed above were positive;

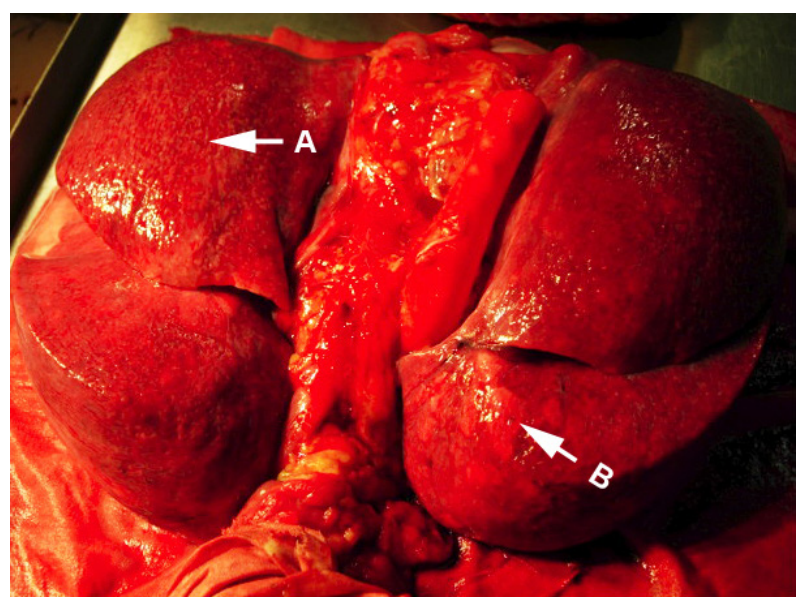

Figure 3. A lung specimen obtained as autopsy showing (A) disseminated miliary foci, and (B) consolidation of miliary densities. 


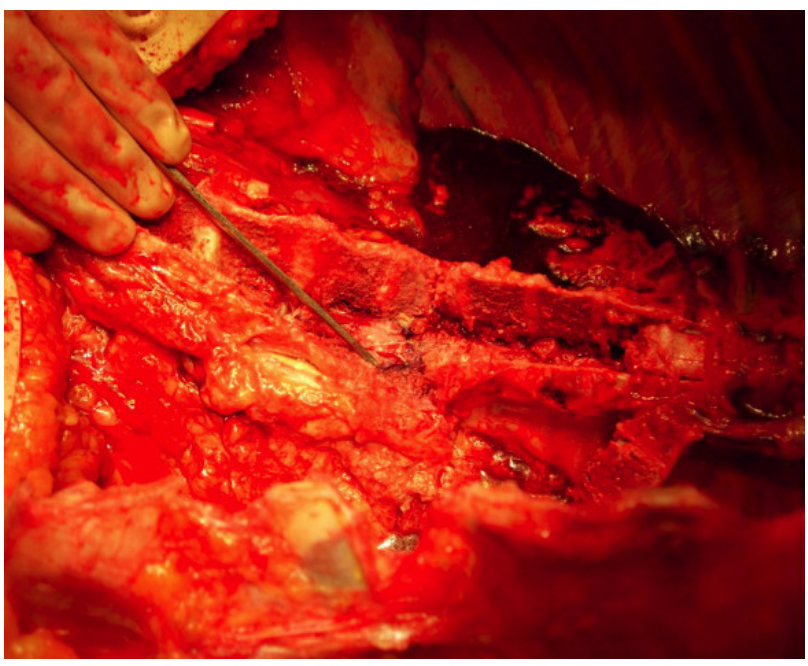

Figure 4. A thoracic spine specimen obtained at autopsy showing the destruction of the T11-12 vertebral bodies with an associated paravertebral abscess.

biopsy cultures grew Mycobacterium tuberculosis colonies. Histology of liver tissue showed steatosis with negative results bymicroscopy and culture. The test for drug susceptibility was not performed. The final pathological diagnosis was generalized TB with involvement of the lung, CNS, spleen, vertebrae, and kidneys complicated by respiratory failure and DIC.

\section{Discussion}

This case presentation describes chronic disseminated tuberculosis in a HIVsero- negative patient. The patient's history suggests that she had active extrapulmonary TB infection prior to her respiratory symptoms. It is likely that the compression fracture of the spine was caused by undiagnosed tuberculous spondylitis, which is the most common location for TB bone involvement. The autopsy confirmed the tuberculous destruction of the vertebral bodies of the thoracic spine with a formation of paravertebral abscess. This chronic and untreated source of TB infection was likely the nidus for the subsequent spread of Mycobacterium tuberculosis to the other organs, including kidneys, spleen, meninges, and lungs. Increas-

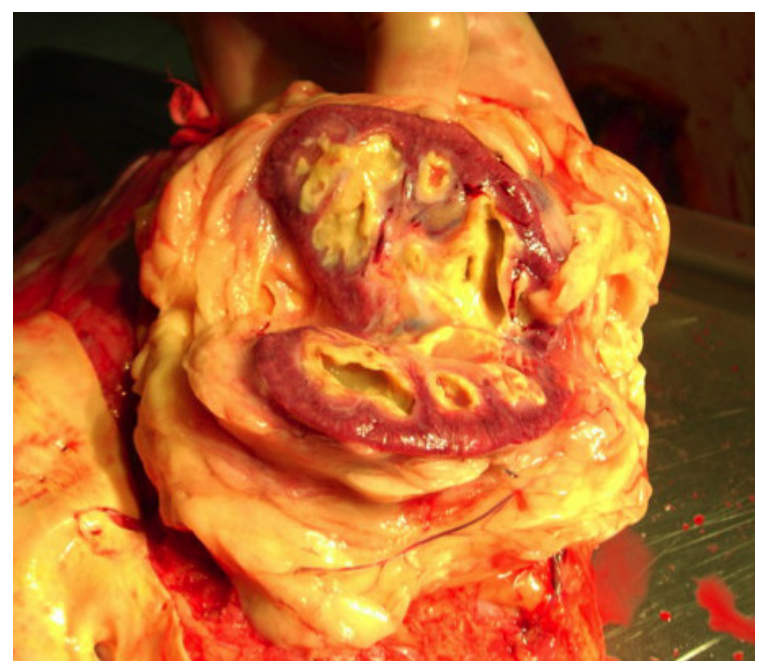

Figure 5. A left kidney specimen obtained at autopsy showing changes consistent with cavernous renal tuberculosis.

ing dyspnea, malaise, and fever prompted the patient to seek treatment. The diagnosis of miliary TB was established by the clinical findings and imaging abnormalities despite the absence of microbiologic confirmation. The patient was placed on the antituberculouschemotherapy, but due to the advanced stage of the infection with DIC and multi-organ failure she died on the third day of admission to the phthisiopulmonology clinic.

The clinical manifestation of miliary TB are non-specific, and this complicates and delays the diagnosis. ${ }^{4,5}$ With symptoms, such as fever, malaise, night sweats, weight loss, and cough, patients commonly receive symptomatic treatment with broadspectrum antibiotics.Visible radiographic changes of the dissemination may take five to eight weeks to occur or may not be present at all, such as in cryptic tuberculosis. ${ }^{6,7}$ CT imaging is usually more informative in this circumstance. In the lungs, hematogenous spread usually leads to asymmetrical and uniform tubercular foci formation. The size, location, and symmetry of the foci vary among patients. Identification of the infection is suggested by "miliary mottling," whose monomorphic elements are 1 to $2 \mathrm{~mm}$ in di- 
ameter and are located uniformly in both lung fields as a "mirror image" with a decreased visualization of the lung marking and a decreased clarity of the lung fields. ${ }^{8}$ Although there are no specific hematological changes with miliary TB, the lymphopenia noted in our patient is not an uncommon abnormality and can be explained by reaction of lymphocytes to the miliary TB infection and migration to tubercular foci. ${ }^{5,9,12}$

The mycobacterial studies are one of the most important components of the diagnosis. Both acid fast stain microscopy and cultures should be performed to confirm the presence of mycobacterial infection. The sensitivity of microscopy ranges from $34 \%$ to $80 \%$, whereas the culture ranges from $80 \%$ to $93 \%$. ${ }^{15}$ Due to the time required to grow the mycobacterial cultures, biopsy of other organs should be considered for the confirmation of the diagnosis. ${ }^{4,10}$ Delayed miliary TB treatment may lead to the development of major complications of disseminated infection, such as DIC and acute respiratory distress syndrome (ARDS)

Our patient developed DIC on the second day of antituberculous chemotherapy. The precise pathophysiologic pathway of triggering DIC in mycobacterial infection is unknown. It is possible that hypoxia with the production of reactive oxygen species and the dissemination of mycobacterial bacilli cause endothelial dysfunction, as in sepsis, with the release of tissue factors triggering the coagulation cascade. Development of DIC is fortunately rare in patients with active tuberculosis but is found in $4.4 \%$ of patients with miliary TB. ${ }^{11,12}$ The treatment of DIC in general and specifically in patients with miliary TB has a poor response and is largely ineffective.

The components of the cell walls of mycobacterial tuberculosis can induce a strong inflammatory response in the form of ARDS with the neutrophil-induced alveolar-capillary membrane damage and the sequestration of fluid into the alveolar space. Similar to DIC, one possible mechanism involves abnormalities in the coagulation system with the formation of intrapulmonary microthrombi.The generalization of mycobacterial infection substantially increases the risk of developing ARDS, and the incidence of ARDS is four times higher in miliary TB than other forms of pulmonary TB. Monitoring patients is crucial to the early detection of this complication, and laboratory tests, such as decreased absolute lymphocyte counts, high alanine aminotransferase levels, and the low serum sodium levels, may help identify these patients. ${ }^{13}$ Mortality rates remain high and can be predicted by the $\mathrm{PaO}_{2} / \mathrm{FiO}_{2}$ rates according to the new proposed "Berlin definition" of ARDS.

This case presentation raises several important issues in contemporary TB diagnosis and management, especially pertinent in the U.S. With declining TB incidence in the U.S. to historically low levels, physicians are becoming progressively less likely to encounter TB in most clinical practices, thereby making suspicion of TB and rapid diagnosis of TB more problematic even for the most common TB presentations. In this case, added confounding features included not just the undiagnosed extrapulmonary (spinal) TB but subsequent TB dissemination in a patient without apparent risk factors for disseminated TB disease making suspicion of the diagnosis even more difficult. Although the miliary pattern on the chest radiograph was characteristic enough to trigger the appropriate evaluation and treatment, it came too late to alter the patient's poor prognosis.

To reduce the risk of late complications with disseminated tuberculosis, a comprehensive patient evaluation is essential as well as awareness of the atypicalpresentation (compared to reactivation pulmonary TB) and course of extrapulmonary TB in order to make a timely diagnosis of extrapulmonary and disseminated TB. All manifestations of active TB, pulmonary and extrapulmonary disease, should be treated according to the standard and easily accessible regimens recommended by the Centers for Disease Control and Prevention (CDC), the American Thoracic Society (ATS), and the World Health Organization (WHO). Patients with miliary TB with late complications have a poor prognosis. 


\section{KEYPOINTS}

1 Miliary tuberculosis has non-specific symptoms and can be a difficult diagnosis; involvement of organs outside the lung parenchyma must be considered.

2. Late stage miliary tuberculosis has a poor prognosis due to the development of disseminated intravascular coagulation and acute respiratory distress syndrome.

3. Clinical suspicion of tuberculosis at the early stage of infection is paramount to management.

Author Affiliation: Tatiana Denega was a resident in Moscow when she cared for this patient. David Griffith is a pulmonary physician at the University of Texas Health Center at Tyler, TX.

Received: $11 / 07 / 2013$

Accepted: $12 / 29 / 2013$

Reviewers: Kenneth Nugent MD, Richard Winn MD

Published electronically: 01/15/2014

Conflict of Interest Disclosures: None

\section{REFERENCES}

1. Yagi O, Kawabe Y, Nagayama N, Shimada M, Kawashima M, Kaneko Y, Ariga H, Ohshima N, Matsui Y, Suzuki J, Masuda K, Tamura A, Nagai H, Akagawa S, Machida K, Kurashima A, Nakajima Y, Yotsumoto H. Bone and joint tuberculosis concurrent with tuberculosis of other organs. Kekkaku. 2007 Jun; 82(6):523529. (Japanese)

2. Simon HB, Weinstein AJ, Pasternak MS, Swartz MN, Kunz LJ. Genitourinary tuberculosis: Clinical features in a general hospital population. The Am J of Med. 1977; 63(3):410-420.
3. Kapoor R, Ansari MS, Mandhani A, Gulia A. Clinical presentation and diagnostic approach in cases of genitourinary tuberculosis. Indian J Urol. 2008 Jul-Sep; 24(3):401-405.

4. Alsoub H, Al Alousi FS. Miliary tuberculosis in Qatar: a review of 32 adult cases. Ann Saudi Med. 2001 Jan-Mar;21(1-2):16-20.

5. Mert A, Bilir M, Tabak F, Ozaras R, Ozturk H, Aki H, Seyhan N, Karayel T, Aktuglu Y. Miliary tuberculosis: clinical manifestation, diagnosis and outcome in 38 adults. Respirology. 2001 Sep; 6(3):217-24.

6. Rosenshtrauch LS, Ribakova NI, Vinner MG(1987). Radiodiagnosis of respiratory system diseases: Physicians Manual (2nd ed.) (pp. 606-609). Moscow: Medicine (Russian)

7. Long R, O’Connor R, Palayew M, Hershfield E, Manfreda J. Disseminated tuberculosis with and without a miliary pattern on chest radiograph: a clinical-pathologic-radiologic correlation. Int J Tuberc Lung Dis. 1997 Feb;1(1):52-58.

8. Proudfoot AT, Akhtar AJ, Douglas AC, Horne NW. Miliary Tuberculosis in Adults. Br Med J. 1969 May 3; 2(5652):273-276.

9. Beck JS, Potts RC, Kardjito T, Grange JM. T4 lymphopenia in patients with active pulmonary tuberculosis. Clin Exp Immunol. 1985 Apr; 60(1):49-54.

10. Dunlap NE, Bass J, Fujiwara P, Hopewell P, Horsburgh RC, Salfinger M, Simone PM. Diagnostic Standards and Classification of Tuberculosis in Adults and Children. American Journal of Respiratory and Critical Care Medicine. 2000; 161(4):13761395.

11. Wang JY, Hsueh PR, Lee LN, Liaw YS, Shau WY, Yang PC, Luh KT. Mycobacterium tuberculosis inducing disseminated intravascular coagulation. Thromb Haemost. 2005 Apr; 93(4):729734.

12. Maartens G, Willcox PA, Solomon BR. Miliary tuberculosis: Rapid diagnosis, hematologic abnormalities, and outcome in 109 treated adults. The American Journal of Medicine. 1990 Sept; Vol. 89(3):291-296.

13. Sharma SK, Mohan A, Banga A, Saha PK, Guntupalli KK. Predictors of development and outcome in patients with acute respiratory distress syndrome due to tuberculosis. The International Journal of Tuberculosis and Lung Disease. 2006 Apr; 10(4):429-435(7).

14. ARDS Definition Task Force, Ranieri VM, Rubenfeld GD, Thompson BT, Ferguson ND, Caldwell E, Fan E, Camporota L, Slutsky AS. Acute respiratory distress syndrome: the Berlin Definition. JAMA. 2012 Jun;307(23):2526-33.

15. Jindal SK. Handbook of Pulmonary and Critical Care Medicine. Jaypee Brothers Medical Publishers. 2012. P59. 